\title{
Concepciones históricas asociadas al concepto de número irracional: Un estudio en libros de texto usados en el grado octavo en Florencia ${ }^{1}$
}

\author{
Historical conceptions associated with the concept of irrational \\ number: A study of textbooks used in the eighth grade in Florence
}

Concepções históricas associadas ao conceito de número irracional: um estudo dos livros didáticos utilizados na oitava série em Florença

Recibido: mayo de 2013

Aprobado: agosto de 2013

Albeiro Giraldo Ospina ${ }^{2}$

\begin{abstract}
Resumen
Este trabajo hace parte de la producción académica desarrollada en el marco de la investigación "Un estudio de los números irracionales en los libros usados en el grado octavo en Florencia", investigación aprobada como tesis de Maestría en Docencia de las Matemáticas de la Universidad Pedagógica Nacional 2008, y realizada a partir de la Dimensión de análisis conceptual, con su respectiva categoría: concepciones históricas, con el propósito de establecer las concepciones históricas que se manifiestan en los libros de texto de matemáticas más usados en el grado octavo en Florencia, Caquetá.

Palabras clave: Otras nociones de Educación Matemática; evolución histórica de conceptos; concepciones históricas; aula; recursos didácticos; libros de texto; matemáticas escolares; números; estructuras numéricas; números irracionales; enseñanza; aprendizaje.
\end{abstract}

\begin{abstract}
This work is part of the academic production developed in the framework of the research "A study of irrational numbers in the textbooks used in the eighth grade in Florence", approved as thesis research Masters in Teaching of Mathematics of the National Pedagogical University 2008, and made from the Dimension of conceptual analysis, with their respective category: historical conceptions, in order to establish historical conceptions that appear in math textbooks most used in the eighth grade in Florencia, Caquetá.
\end{abstract}

Keywords: Other notions of Mathematics Education; historical evolution of concepts, historical concepts, classroom teaching resources, textbooks, school mathematics, numbers, numerical structures, irrational.

1 Artículo de Investigación

2 Universidad de la Amazonia. Colombia. Docente Catedrático de Matemáticas, Director de tesis de Maestría. Docente Matemáticas y Física, Institución Educativa Jorge Eliecer Gaitán, Florencia - Caquetá. Integrante del grupo de investigación Desarrollo Institucional Integrado, línea de investigación Competencias Matemáticas, maestría en Ciencias de la Educación con énfasis en Didáctica de las Matemáticas. Contacto:. albeiro70@gmail.com 


\section{Resumo}

O artigo apresenta os resultados parciais de uma investigação na qual foi proposto um processo de modelagem matemática para o estudo de alguns aspectos da função seno trigonométrico. Ele defende a relevância da modelagem como uma forma de desenvolver outros tipos de representações (modelos) para os conceitos de matemática trabalho específico, que estabelecem relações matemáticas entre as variáveis apresentadas em alguns problemas ambientais, e exigem o desenvolvimento de um modelo para realização bem sucedida. Finalmente, uma metodologia de projeto para trabalhar com simulações de modelagem de processos, a partir do qual emergem conceitos matemáticos subjacentes a funções trigonométricas seno e cosseno.

Palavras-chave: Trigonometria; aprendizagem processos cognitivos, modelagem matemática, simulação, trigonometria.

\section{Introducción}

El estudio del proceso que han seguido los conceptos matemáticos en su formación y en su desarrollo, así como los mecanismos de producción de éstos saberes, y en general conocer las características de la actividad matemática en cada momento o periodo, ha permitido a los investigadores en Didáctica de las Matemáticas determinar concepciones, dificultades y obstáculos ligados a la evolución de una noción matemática.

Ahora bien, teniendo en cuenta que los estudiantes utilizan y trabajan con libros de texto de matemáticas, los cuales se convierten en una herramienta importante en la manipulación del saber, en ellos, se manifiestan concepciones, tal como lo señala Sánchez y Contreras(1988) :"cuando en los manuales se realiza el paso del saber que debe ser enseñado al saber escolar, se manifiestan, entre otros[aspectos], concepciones, obstáculos (epistemológicos y didácticos) y también dificultades; todos ellos relacionados con el saber y cuyo análisis nos puede permitir extraer consecuencias pertinentes acerca de la enseñanza de los conceptos matemáticos" ( $p$. 6) (Subrayado mío).

Claramente, los autores hacen referencia explícita al fenómeno de la transposición didáctica, porque es precisamente en este marco, donde la comprensión de las relaciones del libro de texto con el saber adquiere pleno significado; sin embargo, en este proceso se pueden manifestar múltiples aspectos, entre los cuales están las concepciones.

Sánchez y Contreras (1988) son claros en manifestar que el análisis de las concepciones - en particular las que se manifiestan en los libros de texto-, permiten extraer e inferir consecuencias acerca de la enseñanza, y muy posiblemente del aprendizaje de un concepto matemático; en este caso, el de número irracional, porque de alguna manera las concepciones que manifiestan los profesores y los estudiantes, son influenciadas por las que portan los libros de texto de matemáticas.

Aspectos que también comparte Medina (2001 citado en Mora \& Torres, 2004), al señalar que las concepciones que portan los textos inducen concepciones institucionales acerca de tratamientos didácticos, las cuales pueden ser origen de obstáculos didácticos. Por esto, el análisis de los libros de texto, nos permiten hacer inferencias acerca de la enseñanza y el aprendizaje de los conceptos matemáticos (p.142).

Las concepciones que se identifican en la génesis histórica de un concepto matemático, se denominan concepciones históricas; que como lo señala El Bouazzoui (1988, citado en Sánchez \& Contreras 1998, p. 23), son " aquellas que se identifican en la génesis histórica de una noción matemática, identificadas en una comunidad de matemáticos y, por 
tanto colectivas", es decir, que la evolución histórica de un concepto matemático, en sus etapas y puntos cruciales de su desarrollo (que se caracterizan en términos de las situaciones, representaciones y personajes que aportaron a su consolidación y de la forma como éstos lo manipularon), dio origen a diversas concepciones históricas del objeto matemático.

Dada la importancia y pertinencia de estudiar las concepciones históricas asociadas a un objeto matemático- en particular las que se manifiestan en los libros de texto- para luego extraer y colegir consecuencias acerca de la enseñanza, y muy posiblemente del aprendizaje de un concepto matemático - en este caso el de número irracional-,deseamos saber:

¿Cuáles concepciones históricas asociadas al concepto de número irracional se manifiestan en los libros de texto de matemáticas del grado octavo más utilizado en Florencia, Caquetá?

\section{Referentes teóricos}

Inicialmente se hizo énfasis en el análisis de contenido (Bardín, 1986), como aquel que se centra en las ideas que se expresan y reflejan en un libro de texto teniendo en cuenta su carácter didáctico; donde su fin no es ser estrictamente descriptivo, sino llegar a ser inferencial; por ello es uno de los métodos empleados frecuentemente para la investigación en Educación Matemática, para el análisis de libro de texto. Luego, y teniendo en cuenta que la presentación del análisis de contenido de un libro de texto debe estar acompañada por los focos en que dicho análisis se centra y los criterios que van a utilizarse, se adoptó la perspectiva teórica de Sierra, Gonzáles \& López (1999), quienes en su trabajo de investigación identificaron y describieron estructuradamente los diversos significados de las matemáticas escolares en torno a un concepto matemático presente en los libros de texto de matemáticas, a partir de tres dimensiones de análisis: La dimensión de Análisis conceptual, Análisis didáctico -cognitivo y análisis fenomenológico didáctico. La primera dimensión fue tomada para el análisis de contenido con el que se efectuó este trabajo, y apoyado en las teorías sobre concepciones de Vergnaud (1988, citado en Moreira, 2002), en el estatus de un concepto matemático de Chevallard ${ }^{3}$ (1991), y las concepciones operacional y estructural de un objeto matemático de Sfard $^{4}$ (1991), que me permitieron ver y entender, las diferentes maneras como se presentan los números irracionales en los libros de texto de matemáticas del grado octavo.

En consecuencia y fruto de investigaciones recientes en Educación Matemática en torno al número real y por consultas en textos de historia de las matemáticas, consideramos cinco concepciones históricas relativas a los números irracionales ${ }^{5}$ q que son:

Tratamiento aritmético de los números irracionales; Los números irracionales como magnitudes geométricas (inconmensurables); El número irracional como magnitud continua;: Expresiones algebraicas $y$ analíticas utilizadas para representar números irracionales; y El número irracional como objeto matemático.

\section{Metodología.}

El presente trabajo se enmarcó en el campo de la investigación de la Didáctica de la Matemática, en la línea del análisis de libros de texto, de naturaleza cualitativa con características de orden descriptivo-interpretativo. Tomando en consideración que el contexto es el educativo (el ambiente escolar), nos ubicamos en dos espacios: uno de ellos los

3 Un concepto matemático ha pasado por tres momentos en la historia: Cuando aparece (estatus protomatemático), cuando es usado como herramienta (estatus paramatemático) y cuando es objeto de estudio. Cada paso encierra implícitamente la actividad de la comunidad matemática y las reflexiones respectivas en las diferentes épocas Chevallard (1991).

4 El tratamiento de las nociones matemáticas como objetos abstractos hace referencia a una concepción de tipo estructural, mientras que la descripción de procesos algorítmicos y acciones reflejan una concepción de corte operacional Sfard (1991).

5 Para conocer el completo y detallado estudio historiográfico del número irracional, puede remitirse a la investigación "Un estudio de los números irracionales en los libros usados en el grado octavo en Florencia", aprobada como tesis de Maestría en Docencia de 
conceptos a ser enseñados, y el otro, en las herramientas de apoyo a los procesos de aprendizaje de dichos conceptos.

En cuanto a los conceptos, seleccionamos los números irracionales y, en relación con las herramientas de apoyo, los textos escolares de matemáticas de octavo grado de circulación nacional y utilizados en las instituciones educativas (tanto públicas como privadas), de Florencia (Caquetá, Colombia). Para la selección de dichos textos recurrimos a la identificación de cuáles eran los textos más utilizados por los docentes para la enseñanza y aprendizaje de los números irracionales en dicho grado. Para tal efecto, se realizó una encuesta a 43 docentes de las 14 instituciones educativas oficiales y de las 2 únicas instituciones privadas del área urbana del municipio de Florencia.

Una vez seleccionados los textos, adelantamos un pormenorizado análisis de la Dimensión de análisis conceptual, con su respectiva categoría: concepciones históricas. Seguidamente se elaboró un modelo de rejilla donde se organizó la información de cada texto, registrándose en una matriz de análisis la información relacionada. Luego, a partir de la información obtenida se elaboró una tabla, con el propósito de organizar y presentar los datos, y así, facilitar el respectivo análisis.

\section{Análisis de los datos}

En seguida presentamos la descripción y análisis de los libros de texto ${ }^{6}$ de matemáticas seleccionados, en cuanto a la categoría concepciones históricas:

La tabla 1, pone de relieve que en el $50 \%$ de los textos analizados se manifiesta Tratamiento aritmético de los números irracionales, al igual que El número irracional como magnitud continua; en el 25\% de los textos Expresiones algebraicas y analíticas del número irracional, como El número irracional como objeto matemático y Los números irracionales como magnitudes geométricas inconmensurables, y en dos textos no se hace evidente alguna concepción histórica de número irracional.

Así mismo, en dos textos se manifiesta mayor cantidad de concepciones históricas del número irracional; en dos textos, 2 concepciones históricas, y solamente una concepción histórica en dos textos.

Tabla 1. Concepciones Históricas del número irracional.

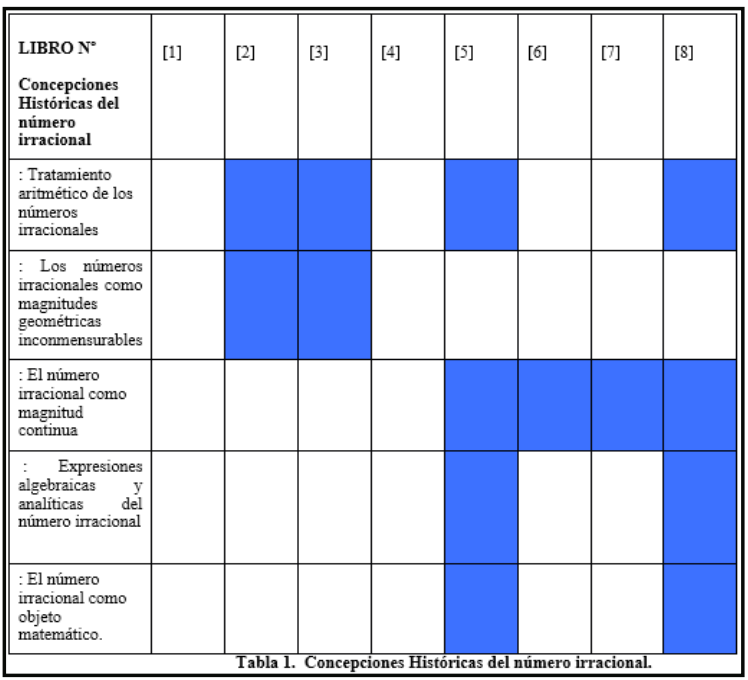

Fuente: Elaboración propia

\section{Conclusiones}

(1)Si un libro de texto de matemáticas no hace manifiesto ningún tipo de concepción histórica relativa al concepto de número irracional, como el caso de los textos [1] y [4], y simplemente se limita a dar una definición formal de él, consideramos que no posibilita un acercamiento a la comprensión (aprendizaje) de este concepto matemático por parte de los estudiantes. Para el libro de texto la presentación formal del concepto, da por sentado el aprendizaje de éste por parte del alumno; sin embargo, investigaciones en Educación matemática han evidenciado que la "definición de un concepto no garantiza la comprensión del concepto" (Tall y Vinner, 1981), ya que la definición formal

6 Los libros de texto de matemáticas del grado octavo analizados, fueron ordenados de acuerdo al año de edición; y son: [1] Londoño, N \& Bedoya, H. (1989). Álgebra y Geometría. Serie matemática progresiva. Editorial Norma; [2] Villegas, M. (2000). Matemática 2000. Editorial Norma; [3] Londoño, N., Guarín, H \& Bedoya, H. (1993). Dimensión Matemática. Editorial Norma; [4] Beltrán, L. 
encapsula las situaciones y procesos que le dan sentido al concepto, ocultando su significación y complejidad;(2) Desde el año 1999, Se detecta que los autores de textos (en particular de los textos [5] y [8]), incluyen en el tratamiento del número irracional las concepciones históricas y como una manera de mostrar la evolución histórica que ha tenido el concepto de número irracional (el proceso que ha seguido en su formación y en su desarrollo), y de acercamiento a su comprensión por parte de los estudiantes.

\section{Referencias}

Bardín, L. (1986). El análisis de contenido. España: Akal.

Chevallard, Y. (1991). La Transposición Didáctica. Del saber sabio al saber enseñado. Argentina: Aique.

Moreira, M. (2002). La teoría de los campos conceptuales de Vergnaud, la enseñanza de las ciencias y la investigación en el área. En: Enseñanza de las Ciencias. Vol. 7. No. 1.
Sánchez, C \& Contreras de la Fuente, A. (1998). Análisis de manuales a través del tratamiento didáctico dado al concepto de límite de una función: una perspectiva desde la noción del obstáculo. En: Rev. Enseñanza de las ciencias. Vol. 16 No. 1, Pp. $73-84$.

Sfard, A. (1991). On the dual nature of mathematical conceptions: Reflections on processes and objects as different sides of the same coin. En: Edgar Alberto Guacaneme (trad.). Educational Studies in mathematics. No. 22. pp. 1-36.

SIierra, M., Gonzales, M \& López, C. (1999). Evolución histórica del concepto de límite funcional en los libros de texto de bachillerato y curso de orientación universitaria (COU): 1940 - 1951. En: Revista Enseñanza de las Ciencias. Vol. 17. No. 3. Pp. 463 - 476.

Tall, D. Y Vinner, S. (1981). Concept Imagen Concept Definition in Mathematics with particular reference te limits and continuity. Educational Studies in Mathematics 12, p.151-169 